\title{
Epistemologisches Reverse Engineering
}

\author{
Oder: Über Techniktheorie(n), Gilbert Simondon und das Echolot
}

[...] eine Maschine zu besitzen heißt nicht, sie zu kennen.

- Gilbert Simondon, $1958^{1}$

\section{○ play}

Im März 1949 schrieb der damals erst jüngst zum Kybernetiker avancierte Max Bense an Hans Paeschke, den Chefredakteur der Zeitschrift Merkur, über einen der ersten vollelektronischen universalen Digitalcomputer: Den ENIAC, den Electronic Numerical Integrator And Calculator. Bense wollte Paeschke dazu bewegen, in der Zeitschrift über, Elektronenhirne ${ }^{2}$ im Sinne der Kybernetik zu publizieren, die vermeintlich die potenzielle Äquivalenz maschineller und humaner Nachrichtenübermittlung aufzeigen würden. Die Überlegungen Benses wurden schließlich 1951 im Merkur publiziert. ${ }^{3}$ Bense schrieb:

Natürlich interessiert Sie die Eniac besonders. Ich verrate Ihnen, daß diese Maschine einen Raum mit 150 m Kantenlänge einnimmt, daß sie mit 15000 Röhren (Radioröhren) arbeitet, auf 150 Kilowatt läuft, 30 Tonnen wiegt und 320 Kilometer Draht aufweist. C’est tout. - Die jüngsten elektronengesteuerten Maschinen [...] arbeiten mit einem Ja-Nein Prinzip, machen also vom Grundsatz der chrysippischen und russellschen Aussagenlogik Gebrauch, danach eine Aussage ein Gebilde ist, das die Eigenschaft hat, entweder wahr oder falsch zu sein. D. h. logische Prinzipien sind in technische umgesetzt worden $!^{4}$

Neben der Implementierung von Logik in Technik, um Techno-Logie und damit eine Maschinisierung des Denkens zu erzeugen, sind es die massiven räumlichen Ausmaße des ENIAC, die Benses Beschreibung besonders auszeichnen. Wie Jennifer Light geschildert hat, bedeutete dies zudem für die ausschließlich (!) weiblichen Programmiererinnen des ENIAC, ${ }^{5}$ dass sie sich im technischen Objekt befanden, während sie ihn durch das Stecken von Kabelverbindungen programmierten, statt durch ein Interface

1 Simondon (2012 [1958]), 232.

2 Hierzu auch Pias 2004.

3 Bense (1998 [1951]).

4 Zit. n. Hartmann 2009.

5 Deren Namen von der Computergeschichte gern vergessen werden, obgleich sie dem ,Elektronenhirn'schließlich ihre Berufsbezeichnung vererben sollten: Computer. 
mit dem Computer zu kommunizieren. ${ }^{6}$ Heute, im Jahr 2020, befinden sich Menschen allerdings - wenngleich fast fortwährend in Maschinenparks und medialen Welten eher selten in technischen Medien. Wer wissen will, wie es um ihre innere Struktur steht, um ihre embedded systems, der kommt um ihr Öffnen nicht umher.

Doch was, wenn das technische Medium nicht geöffnet werden kann (man denke an heutige Smartphones, bei denen nicht einmal ein Akku gewechselt werden kann)? Wenn durch das Öffnen wiederum allein schwarze Kästchen sichtbar werden (bei hochtechnischen Medien stößt die Phänomenologie an ihre Grenzen, das Rechnen des Mediums ist unsichtbar)? Oder nicht zuletzt was, wenn das technische Objekt ein historisches ist und als materiales Artefakt abwesend, d. h. der Öffnung oder direkten Untersuchung unzugänglich? Und überhaupt, was wird in der Technikforschung eigentlich mit einem Öffnen von Black Boxes in unterschiedlichen Ansätzen gemeint? ${ }^{7}$

Technische Medien als komplexe, verschlossene, eingekapselte und der materiellen Öffnung durch Laien tendenziell nicht andienende „Prozessarchitekturen“ ${ }^{\text {( } 8}$ bestimmen auch 2020 noch sichtbar oder verborgen, mikro- oder makro-präsent „unsere Lage“. ${ }^{9}$ Wie kann sich die Medienwissenschaft bzw. die Science and Technology Studies ihren Untersuchungsgegenständen und den medienbestimmten ,Lagen“ nähern? Generell lassen sich dafür im Feld aktuell beziehungsweise verstärkt seit den 1990er Jahren methodisch - neben anderen vielversprechenden Ansätzen - dominant (und dominierend) zwei diametral zueinander stehende Zugänge identifizieren. Einmal ist dies ein tendenziell eher materialistisch orientierter Ansatz, wie er im Zusammenhang mit Friedrich Kittlers kontrovers diskutierter Forschung als technikdeterministisch ${ }^{10}$ apostrophiert wird. Andererseits ist dies ein eher soziologisch-anthropologischer Ansatz, wie er beeinflusst von der Actor-Network-Theory (ANT) und der Kulturtechnik-Forschung prominent durch Erhard Schüttpelz' Forderung nach einer „medienanthropologischen Kehre der Kulturtechniken“11 im Jahr 2006 reanimiert wurde, sich vornehmlich am Begriff der „Operationskette“ abarbeitet und - ebenso wie Kittlers Ansatz - in der Kritik steht.

6 Light 1999.

7 Eine Suche nach „opening the black box of“ (in Anführungszeichen) bringt bei Google.de rund 184.000 Treffer, die sich mit so unterschiedlichen Themen beschäftigen wie „opening the black box of Editorship“, „of Energy Modelling“, „of Deep Neural Networks“, „of Innovation“, „of Relevance“, „of Migration“, ...

8 Diesen Begriff entlehne ich Jany 2015.

9 Kittler 1986, 3.

10 Überhaupt erscheint mir fragwürdig, warum der Begriff des „Technikdeterministischen“ negativ konnotiert ist. Dass (Medien-)Technik praktische Handlungsmöglichkeiten, psychische Denkstrukturen und die Formatierung des Sozialen bestimmt, festlegt und begrenzt (das ist die Bedeutung des Begriffs „determiniert“), erscheint mir vielmehr ein Fakt als eine Unterstellung zu sein. Außerdem schließt der Begriff die gegenteilige Richtung - dass potenziell auch Praktiken, Denkstrukturen und soziale Kooperationen wiederum Techniken determinieren - nicht aus.

11 Schüttpelz 2006. 
Zunächst wird der Beitrag diese beiden Pole und ihre Argumentationen nachzeichnen („rewind“) und anschließend eine dritte Methodik darlegen (,re-play“), die, wie gezeigt werden kann, eventuell im Stande ist, beide vermeintlich dualistischen Ansätze produktiv miteinander zu verschalten. Wie dies praktisch an einem medienhistorischen Artefakt geschehen kann, wird kursorisch in einem dritten Schritt eingelöst („reverse“), der sich dem ersten funktionalen Echolot, des deutschen Alexander Behm, widmet, gemäß der Prämisse eines epistemologischen Reverse Engineering (RE). Epistemologisches RE, so mein begrifflicher Vorschlag, könnte als methodische Praxis einige Antworten auf die oben gestellten Fragen liefern. Ob dies als Medienarchäologie, Techniktheorie oder -geschichte, historische Praxeologie oder gar Medienphilosophie gelabelt werden könnte, ist dabei gar nicht so entscheidend, wodurch die gängigen Fallstricke der diskursiven Verortung umgangen werden können.

Das RE versteht sich gemeinhin als eine Dekonstruktion technischer Objekte oder Softwares, um ihre innere Architektur und Systematik freizulegen und damit aus einer schwarzen Kiste einen grundlegenden Konstruktionsplan dieser erstellen zu können oder, in Bezug auf Software, einen Quellcode zu gewinnen. Das zu untersuchende Objekt soll dabei funktional möglichst exakt abgebildet, statt ein analoges Objekt entwickelt werden, das dieselben Ergebnisse erzielt, wodurch sich das RE vom Black Box-Konzept der Kybernetik scheidet, nach welchem - wie von Ross Ashby in seiner Introduction to Cybernetics dargelegt - allein empirisch In- und Output-Größen eines technischen oder biologischen Dings gemessen werden. ${ }^{12}$ Da das Behm'sche Echolot allenfalls noch in Museen in Vitrinen zugänglich ist, muss dies zum einen theoretisch geschehen, andererseits wird mit der theoretischen Öffnung der Black Box seine technik- und wissensgeschichtliche Bedingung freigelegt werden. Das epistemologische RE versteht sich in diesem Sinne als ein produktiver Rückschritt bzw. als ein Zurückschreiten (reversing). Das Echolot tritt hier nicht als materialer Ausgangspunkt einer in die Zukunft schreitenden Mediengeschichte oder als Glied einer Operationskette auf, sondern es markiert zunächst einmal einen Endpunkt, der auf seine Vorgeschichte und vorgelagerten Praktiken hin befragt werden kann.

Vorab muss dazu angemerkt werden, dass eine solche Perspektivierung der Black Box (in diesem Fall die Hülle eines Echolots) als das, was als störend für das Verstehen ihres Inneren ist, wie bspw. von der Medienarchäologie vertreten, das Potenzial von Boxes als materielle und multifunktionale Kapseln verschweigt. In den seltensten Fällen sind Gehäuse allein Grenzflächen zwischen einem wie auch immer gelagerten Innenraum und einem Außenraum, sondern funktionaler Bestandteil des Gesamtgefüges (man denke an Touchscreens, Häuserwände, Computergehäuse, Schalentiere). Das Verständnis des Gehäuses als abzutragendes Blendwerk ,Black Boxt‘ den design-ästhetischen und funktionalen Wert der Box; bspw. ist jedes Interface eine operative Grenzfläche der Kommunikation zwischen zwei Systemen. ${ }^{13}$

12 Ashby 1956, 86-117.

13 Siehe hierzu auch Christina Bartz et al. 2017. 


\section{(1) rewind}

Die methodische Frage, wie technische Objekte methodisch zu analysieren seien, ist sicher genauso alt wie eine institutionalisierte Technik- und Medienwissenschaft selbst. Mein Ausgangspunkt, die beiden (vermeintlich) unvereinbaren Pole techniktheoretischer Reflexion darzulegen, sind im Folgenden zwei Texte, die um das Jahr 2000 herum entstanden sind: Hartmut Winklers Aufsatz „Die prekäre Rolle der Technik“ (1999) sowie Henning Schmidgens Aufsatz „Der Psychologe der Maschinen“ (2001). Generell lassen sich aktuell, so beide Autoren vor knapp zwei Jahrzehnten, zwei fundamental verschiedene Ansätze im Feld identifizieren, Medien- oder allgemeiner: Technikforschung zu betreiben. Dies ist selbstredend stilisiert und eher eine Zuschreibung der jeweiligen Gegenseite, die idealtypisch so nur selten anzutreffen ist, dennoch halte ich diese Differenzierung in zwei Hauptstränge für tendenziell zutreffend.

Einmal handelt es sich dabei um einen eher ,technikzentrierten` Ansatz, wie er prominent durch Friedrich Kittlers Paradigma der Austreibung des Geistes aus den Geisteswissenschaften zugunsten medientechn(olog)ischer Aprioris formuliert wurde: „Medien ,definieren, was wirklich ist““; ${ }^{14}$ „Nur was schaltbar ist, ist überhaupt“; ${ }^{15}$ „Von Leuten gibt es immer nur das, was Medien speichern und weitergeben können“; ${ }^{16}$ „Der sogenannte Mensch zerfällt in Physiologie und Nachrichtentechnik. “17 Technische Medien können in diesem Sinne auf gewisse Operativitäten reduziert und formal-logisch abgebildet werden. In Ansätzen, die daran anschließen, erscheint das Artefakt als Vergegenständlichung von Wissen oder Logik, als in Materie existierende Operationen. Medientechniken sind in dieser Lesart nicht gleichzusetzen mit Kulturtechniken. Damit einher geht eine Hinwendung zur Materialität der Kommunikation; im Sinne Michel Foucaults zur Bedingung dessen, was in einer Kultur aussagbar, nämlich speicher-, prozessier-, übertragbar oder überhaupt ,wiss-bar` ist. Mithin: es geht ums Große, ums Ganze, um medieninduzierte Eskalationen, die den Menschen schließlich hinter sich lassen, oder mit Rudolf Maresch kommentiert:

Medientechnologien, die Muster der Wahrnehmung und Erfahrung vorgeben, nicht Reflexion und Selbstbewußtsein, legen nämlich die Normen und Standards fest, die einer existierenden Kultur die Auswahl, Speicherung und Übertragung relevanter Daten erlauben. [...] Nach diesen materiellen, technischen und historischen Ermöglichungsbedingungen gesellschaftlicher Kommunikationen zu fragen, bedeutet, jene medientechnischen Blindheiten zu entziffern, die Wissen und Macht jahrtausendelang kennzeichnete. ${ }^{18}$

14 Kittler 1986, 10. Kittler zitiert Bolz 1987, 34 (es handelt sich dabei um einen Beitrag in einem Sammelband, den Kittler mitherausgegeben hat - dadurch kann er , aus der Zukunft zitieren').

15 Kittler 1990, 363.

16 Kittler 1986, 5.

17 Kittler 1986, 29.

18 Maresch 1996. 
Bei aller zum Teil berechtigter Kritik an diesem Ansatz darf allerdings nicht vergessen werden, dass Kittler bei allem ,Technikdeterminismus' durchaus auf sich verändernde Praktiken fokussierte, wie beispielsweise die sich ändernden Praktiken der Literalitätserziehung um 1800. Oder mit den Worten des Betreffenden: Unsere medienbestimmte Lage verdient „(trotzdem oder deshalb) eine Beschreibung.“19

Demgegenüber stehen eher anthropologisch ausgerichtete Ansätze von Technikforschung, die ihren analytischen Ausgangspunkt am Menschen ansetzen. Anthropologisch sind diese Ansätze, weil sie am Menschen als Subjekt beginnen und an diesem festhalten und nicht in den Medientechniken (und medialen Aprioris), sondern in den Praktiken den Grund medienkultureller und -gesellschaftlicher Veränderungen sehen. „Anthropologisch in diesem Sinne wären nahezu alle Medientheorien bis zum historischen Bruch bei McLuhan, der neben Benjamin als Hauptzeuge der theoretischen Wende in Anspruch genommen wird; anthropologisch wären die Mediensoziologie, Medienpsychologie und -pädagogik, aber auch und vor allem die Tradition der Ideologiekritik“. ${ }^{20}$ Doch das reicht nicht aus, denn alle von der ANT beeinflussten Ansätze subsumieren sich auch unter die anthropologisch ausgerichtete Medienforschung. Vornehmlich und teilweise buchstäblich beginnen diese ihre Untersuchungen ,vor Ort', in situ, bei der Mikroebene und nicht, von oben'. Was ist damit gemeint? Die situierte Medienforschung fokussiert auf die lokalen Sozialbeziehungen und tatsächlich anwesenden Aktanten - also menschliche und nicht-menschliche Akteure - und Netzwerke bzw. auf die Beziehung zwischen Menschen und (technischen) Medien. ${ }^{21}$ Diese sind empirisch (durch Beobachtung, Feldforschung, Interviews usw.) erforschbar, also mit Methoden der Soziologie, weshalb diesbezüglich von einem practice turn in der Medienwissenschaft gesprochen werden kann: „Fragen von Medienspezifik und -differenz sind so nicht mehr allein auf der Ebene ästhetischer und oder technischer Charakteristika eines Mediums beantwortbar, sondern erfordern auf Praktiken gerichtete methodische und begriffliche Zugänge.“22

Vereinfacht gesagt: Erstere Ansätze setzen am Objekt an, das beschrieben, historisch und genealogisch rekonstruiert werden kann, das Lagen bestimmt, soziale Praktiken (re-)konfiguriert, das als materiales Artefakt geöffnet werden kann. Zweitere Ansätze setzen komplementär am Subjekt an, dessen Verhalten und Mediennutzung beobachtbar und das ein soziales Wesen ist, welches zugleich die Objekte erst verfertigt. Technik ist hier das Resultat außertechnischer Prozesse und kann entsprechend auf Praktiken zurückgeführt werden (was nicht zu verwechseln ist mit Martin Heideggers Aussage, das Wesen der Technik sei nichts Technisches). Mit Hartmut Winkler zusammengefasst fragen zweitere Ansätze nach dem Umschlagpunkt von

19 Kittler 1986, 3.

20 Winkler 1999, 225.

$21 \mathrm{Zu}$ diesem letzten Punkt siehe bspw. Thielmann/Schüttpelz 2013.

22 Gießmann 2018, 95. Zur praxistheoretischen Ausrichtung von Medienforschung siehe auch Thielmann 2012. 
Diskurs in Struktur, erstere nach dem Wiederumschlagen von Struktur in Diskurs. ${ }^{23}$ Oder mit Henning Schmidgen:

Die eine Theorie impliziert eine formalisierte Auffassung des technischen Objekts, die analytisch-kombinatorisch oder, in neuerer Zeit, informationstheoretisch unterbaut ist. In dieser Theorie erscheint die Maschine als eine Vergegenständlichung symbolischer oder logischer Ausdrücke. Die andere Theorie ist ethnologisch oder anthropologisch ausgerichtet und erfasst das technische Objekt als eine Art Ganzheit, die in ihrem Bestand, ihrer Entwicklung und Auswirkung empirisch-deskriptiv zu untersuchen ist. So wie bei Lacan erscheinen in der ersten Theorie Maschinen als Verkörperungen von Wissen, die zurückgeführt werden können auf Kombinationen elementarer Bestandteile. Die Kombination dieser Teile oder Elemente folgt, so wird dabei angenommen, einer Logik oder Grammatik, die allgemein gültig ist. In der zweiten Theorie erscheinen Maschinen hingegen wie bei Latour als Körper (als Dinge oder Wesen), die in ihrer Form, Entstehung und Wirkung von örtlich definierten Sozialbeziehungen abhängen und die daher im Hinblick auf solche Beziehungen zu beschreiben sind. ${ }^{24}$

Und obgleich der Begriff der „Black Box“ (etymologisch selbst eine Black Box, insofern der Entstehungskontext des Begriffs nicht geklärt ist ${ }^{25}$ ) in beiden Ansätzen prominent vertreten ist, referieren sie dabei auf je Unterschiedliches. Wenn es nun um das Öffnen von Black Boxes geht, haben wir es entsprechend bei beiden (reduktionistisch dargestellten) Ansätzen mit zwei unterschiedlich gelagerten Analysesphären zu tun: Einmal mit materiellen Dingen, die geöffnet, verstanden, nachvollzogen etc. werden können - die Black Box als zu öffnende und damit zu weißelnde Kiste - und andererseits um das Black Boxing, einem sozialen Handeln bzw. einem Prozess, einer „Vergehäusung“ im Sinne Heike Webers, ${ }^{26}$ der in einem Schwärzeln von Vorgängen, aber eben auch Dingen resultiert, mithin eine Praxis. Dies wird auch bei Bruno Latour, der Schlüsselfigur für die Ausrichtung der ANT, explizit, der - obgleich er über die „Black Box Overhead-Projektor“ schreibt, die wiederum aus diversen Black Boxes besteht - mit dem Begriff vornehmlich auf eine Praktik referiert. In Pandora's Hope: Essays on the Reality of Science Studies gibt Bruno Latour im Glossar eine präzise Definition dessen, was Black Boxing für die ANT bedeutet:

An expression from the sociology of science that refers to the way scientific and technical work is made invisible by its own success. When a machine runs efficiently, when a matter of fact is settled, one need focus only on its inputs and outputs and not on its internal complexity. Thus, paradoxically, the more science and technology succeed, the more opaque and obscure they become. ${ }^{27}$

23 Winkler 1999, 229.

24 Schmidgen 2001, 266-267.

25 Eine (mögliche) Herkunft des Begriffs gibt von Hilgers 2011. Eine andere Vater in diesem Band.

26 Weber 2017, 120. Kursivierung C. B.

27 Latour 1999, 302. Kursivierung C. B. 
Es geht demnach nur augenscheinlich wie dem ,technikzentrierten` Ansatz um das Öffnen von Artefakten und um ihre innere Struktur bzw. Implementierung von Logik in Materie, sondern vielmehr um den soziologisch/ethnologisch untersuchbaren Prozess der Unsichtbarmachung und -werdung wissenschaftlicher Arbeit und technischer Operativität durch ihr Gelingen. „Vielleicht darf man sogar sagen, der Soziologie [der Technik sowie der Medienanthropologie, C. B.] gehe es hauptsächlich um die technisch vermittelten sozialen Praktiken, wobei die technischen Artefakte, als ein Teil dieser Praktiken, auch von Interesse sind. “28 Diese Fokussierung auf Praktiken statt Artefakte geht mit einer Akzentverschiebung von den Substantiven auf die Verben einher, wie sie wenige Jahre nachdem die oben genannten Aufsätze von Schmidgen und Winkler erschienen sind, paradigmatisch in Erhard Schüttpelz' Aufsatz zur „medienanthropologischen Kehre“ von 2006 radikalisiert wurde - und Schüttpelz damit die Frage nach einer etwaigen produktiven Verschränkung beider oben genannten (idealtypischen) Ansätze klar zugunsten eines soziologischen/praxeologischen/anthropologischen Ansatzes entschied. Wenn Henning Schmidgen bereits 2001 konstatierte, dass bisher wenig zur Vermittlung zwischen beiden geschilderten Ansätzen der Technikforschung unternommen wurde, könnte man entsprechend (bewusst plakativ bzw. sogar provokativ) behaupten, die Lage habe sich auch in den darauffolgenden Jahren nicht geändert. Denn Schüttpelz zufolge habe es der Medien- als Kulturtechnikforschung programmatisch darum zu gehen, „vor die Reifizierung [d. $h$. Vergegenständlichung, C. B.] von Apparaten und Substantiven zurückzugreifen, um einen Zugriff auf die Verben und Operationen zu ermöglichen, aus denen die Substantive und Artefakte erst hervorgegangen sind“. ${ }^{29}$ Damit fordert er eben jene oben erwähnte Fokussierung auf Praktiken und Handlungsverkettungen statt Materialitäten radikal ein. Ebenso praxisbezogen heißt es bei Bernhard Siegert, den Schüttpelz zitiert: „Charakterisiert werden kann der methodische Ansatz auf dem Gebiet der Kulturtechniken durch die Betonung des Praxis-Aspekts in der medienhistorischen Analyse: Medien werden dann als Kulturtechniken beschreibbar, wenn die Praktiken rekonstruiert werden, in die sie eingebunden sind, die sie konfigurieren oder die sie konstitutiv hervorbringen. “30 Letztlich soll damit einer vermeintlichen ,Praxisvergessenheit‘ techniknaher Ansätze Abhilfe geschafft werden. Zudem hat die Forderung von Schüttpelz ein methodologisches Substrat: Praktiken sind beobachtbar und infolgedessen können Methoden aus der Anthropologie, Soziologie, Praxeologie zur medienwissenschaftlichen Forschung beitragen, und in dieser Lesart ist die Forderung am radikalsten, Medienwissenschaft würde damit zur Medienanthropologie. Schüttpelz behauptet dementsprechend eine ,heuristische, historische und praktische ,Priorität

28 Schmidgen 2001, 272.

29 Schüttpelz 2006, 87.

30 Schüttpelz 2006, 87. Kursivierung C. B. Zur Betonung des Praxisaspekts siehe auch Gießmann 2018, 96 (Anm.22), „Medien lassen sich einerseits als etwas begreifen, das fortwährend wechselseitig in Aktion neu hergestellt wird, als eine Praxis, die immer im Werden begriffen ist.“ 
der Operationsketten` vor den durch sie gestalteten Größen, und zwar vor allen beteiligten Größen, seien diese Artefakte, Personen und Zeichen, oder technische Objekte, Praktiken und Wissensformen." ${ }^{31}$ Unter dem Begriff der Operationskette formiert sich eine Methode, die, so Schüttpelz 2010,

die Aufmerksamkeit auf die sukzessive Verknüpfung und Koordination der Tätigkeiten richtet, die einer Verrichtung zugrunde liegen. Die Operationskette besagt, dass sich die technische Aufmerksamkeit der Beteiligten an dieser sukzessiven Verknüpfung von Operationen orientiert und dass sich auch die Beschreibung - und die Techniktheorie - erst einmal an der Handlungsverknüpfung orientieren sollte und nicht an den Werkzeugen, am Resultat oder an einem einzelnen Schritt. ${ }^{32}$

Bei aller Nachvollziehbarkeit der Forderung nach einer medienanthropologischen Kehre in der Medienforschung läuft diese Gefahr, als Rechtfertigung zu dienen, sich gerade nicht mehr mit den ,harten“ medienwissenschaftlich relevanten Vorgängen auseinanderzusetzen und (technische) Medien allein noch äußerlich und das heißt: als Black Boxes zu betrachten und sie damit in eine Kategorie mit schlichten Werkzeugen zu stellen. Denn, was bei einer medienanthropologischen Ausrichtung nicht erstaunen sollte, nur scheinbar stehen die Medien im Fokus, eigentlich aber die menschlichen Akteure und deren Handlungsverkettungen. ${ }^{33}$ Allerdings ist zwar Mediengebrauch beobachtbar - und somit auch anthropologisch-soziologisch erforschbar -, technische Medien selbst allerdings sind nicht beobachtbar bzw. höchstens ihre Oberflächlichkeit, ihre ,Boxes‘, weshalb das tatsächliche Operieren technischer Medien die buchstäbliche Black Box medienanthropologischer Forschung ist. Daher mein Einwand: Die Argumentation von Schüttpelz wird brüchig, wenn eine in der Kulturtechnikforschung - ich möchte behaupten zu Unrecht - vorgenommene Gleichsetzung wieder zugunsten einer Unterscheidung aufgehoben wird. Konkret geht es um die Gleichsetzung von Werkzeugen mit technischen Medien, oder anders: Es geht um die Degradierung technischer Medien zu schlichten Werkzeugen. Das soll nicht heißen, dass technische Medien nicht als Werkzeuge verwendet werden können, nur dass sie dann eben keine Medien mehr sind. Wenn ich mit meinem Laptop einen Nagel in die Wand hämmere, ist mein Laptop nicht länger technisches Medium, sondern tritt als Hammer auf, wenngleich seine materielle Eignung zum Hammer zu wünschen übrig lässt und aus dieser Exaption, Zweckentfremdung, insofern nichts radikal Neues entsteht. ${ }^{34}$ Diese Gleichsetzung von technischen Medien mit Werkzeugen in Bezug auf das Konzept der Operationskette findet sich programmatisch beispielsweise bei Harun Maye: „Werkzeuge und Medien existieren nur in den Gesten

31 Schüttpelz 2006, 91.

32 Schüttpelz 2010, 111.

33 Der Vollständigkeit halber bleibt festzuhalten, dass Schüttpelz’ Agenda auch nicht auf die Explikation von (technischen) Medien abzielt. Es geht ihm um „das Verhältnis von Kulturen, Medien und Techniken“ und seine Bestimmung. (Schüttpelz 2006, 90).

34 Zum Thema der ,Exaption‘ siehe auch den Beitrag von Oliver Schlaudt in diesem Band. 
und Operationsketten, in denen sie technisch wirksam werden. Der Mensch verliert seine ausgezeichnete Position und wird zum Glied in einer Kette, zum ,Servomechanismus' seiner Techniken, die er von sich abgespalten und in technische Medien ausgelagert hat. “35 Worin, ließe sich nun fragen, besteht die Differenz zwischen Werkzeugen und Medien? Werkzeuge, im Unterschied zu Medien, daran erinnert Wolfgang Ernst in Rückgriff auf John Dewey, wirken auf Materialien ein, „[w] as ein Material [dahingegen] zu einem Medium macht, ist der Umstand, daß es als Mittel benutzt wird, eine Bedeutung zum Ausdruck zu bringen, die anderer Art ist als die, die es kraft seiner puren physischen Existenz besitzt" - als(o) Information. “36 Ergo: Werkzeug $\neq M e$ dium. André Leroi-Gourhan, auf den sich Schüttpelz bezieht und der den Begriff der Operationskette popularisierte, ${ }^{37}$ beschränkt seine diesbezüglichen Überlegungen im Übrigen sorgsam auf Werkzeuge, wenn er schreibt: „Tatsächlich existiert das Werkzeug nur im Operationszyklus“. ${ }^{38}$ Ob dies für ihn auch für technische Medien - wie die ,Elektronengehirne‘, über die er schrieb - gelte, lässt sein Werk offen.

Und es stimmt. Technikzentrierte Analysen und die klassische Technikgeschichte tendieren dazu, den kritischen Anteil menschlicher Praktik für das Gelingen von Medientechnik nicht zu reflektieren. So ist beispielsweise die frühe Technikgeschichte des deutschen Radar bestens aufgearbeitet, ${ }^{39}$ ihre praxeologische Untersuchung stand bisher allerdings aus, und wie sich herausstellte, war es gerade der Anteil menschlicher Arbeit, der Radar als Medientechnik überhaupt erfolgreich werden ließ. ${ }^{40}$ Dennoch steht die Forderung, Technikforschung anthropologisch am Konzept einer Vorgängigkeit der Operationsketten auszurichten in der Kritik, da diese - so ließe sich zusammenfassen - von medientechnische Aspekten eher ablenkt, wie von Winkler ${ }^{41}$ oder Till Heilmann ${ }^{42}$ formuliert. Diese Letztbegründung von Technik- als

35 Maye 2010, 132-133. Kursivierung C. B. Dass ein Hammer allein im Akt des Hämmerns den Status eines Hammers innehat, mag sein. Auch ein Radio ist nur Radio im Vollzug (wenn es elektromagnetische Wellen empfängt), auch ein Computer hat den Status eines Computers, wenn er rechnet etc. dafür ist die Anwesenheit eines Menschen jedoch irrelevant.

36 Ernst 2008, 158, im Binnenzitat Dewey (1988 [1934]), 234.

37 Leroi-Gourhan 1980, insb. Kap. VIII, S. 273-295. Ursprünglich geht der Begriff auf Marcel Mauss (1947) zurück.

38 Leroi-Gourhan 1980, 296. Allerdings führt Leroi-Gourhan dies im Kontext des Verhältnisses von Werkzeug und Ethnologie an; die Betonung des Gebrauchsaspekts kann folglich durch diese Verhältnismäßigkeit erklärt werden. Kurz darauf schreibt er, „so existiert das Werkzeug real nur in der Geste, in der es technisch wirksam wird“ (Leroi-Gourhan 1980, 296), was wiederum zwei Schlüsse zuließe: 1.) Dass das Werkzeug auch außerhalb der Geste existiert, nur nicht wirksam wird und 2.) dass technische Medien auch ohne Zutun des Menschen wirksam würden, da sie nicht einer Geste bedürfen (ein weiterer Unterschied zum Werkzeug).

39 Siehe bspw. die umfangreichen Arbeiten von Fritz Trenkle: Trenkle 1979; Trenkle 1982a; Trenkle 1982b; Trenkle 1987.

40 Borbach/Thielmann 2019.

41 Winkler 2015, 25.

42 Heilmann 2016; 2017. 
Praxisforschung, die Rückführung medientechnischer Artefakte auf den ,archimedischen Fluchtpunkt‘ vorgelagerter Praktiken bringe es mit sich, „dass in praxeologischen Untersuchungen an der analytischen Leerstelle, welche der kategoriale Ausschluss des Technisch-Artefaktischen gelassen hat, häufig die lang verschmähte Figur des handelnden Subjekts wiederkehrt““. ${ }^{43}$ Die Eigensinnigkeit technischer Medien gerät dabei allzu leicht aus dem Blick bzw. dient eine solche Praxisfokussierung medienwissenschaftlicher Forschung allzu schnell als Rechtfertigung, sich gerade nicht mehr mit den spezifischen Materialitäten, Schaltkreisen, Blockschaltbildern, Softwares $\mathrm{zu}$ beschäftigen. Und bei allem Verständnis für eine praxeologische Begründung der Technikforschung, die Behauptung einer historischen Priorität der Operationskette in dem Sinne, dass ,alle technischen Gegenstände hergestellt werden [müssen], und daher beinhalten sie - dingbiografisch gedacht - eine historische Priorität der Operationskette ihrer Herstellung vor dem Produkt“, kann kaum als medienwissenschaftlich fundiertes Argument gelten. Denn zwar mag in Ashbys „Black Box“-Kapitel (vgl. Anm.12) ein technisches Gerät buchstäblich vom Himmel fallen, nämlich ein außerirdisches aus einem UFO, diesseits aller science fiction würde aber wohl niemand ernsthaft bezweifeln wollen, dass technische Dinge von Akteuren auf dieser Welt hergestellt werden müssen.

Zusammengefasst: Es scheint so, als ob die oben dargestellten diametralen Ansätze, Technikforschung zu betreiben, wie durch die Auseinandersetzung zwischen Schüttpelz und Heilmann belegt, weiterhin im Diskurs fortbestehen. Auf der einen Seite stehen Ansätze, die auf Software, (Quell-)Codes, Hardware, Materialität usw. und damit die ,harten' medienwissenschaftlichen Vorgänge insistieren. Auf der anderen Seite stehen Ansätze, die auf handelnde Subjekte und deren (Medien-)Praktiken fokussieren. Allerdings existiert bereits seit den 1950er Jahren ein technikphilosophischer Ansatz, der die beiden unvermittelt gegenüberstehenden Ansätze verbindet, insofern er ihre Differenzen unterläuft. Wie bereits von Schmidgen dargelegt, könnte sich eine zukünftige Technikforschung produktiv an ihr ausrichten. Konkret handelt es sich dabei um die Technikphilosophie von Gilbert Simondon (1924-1989) und sein Hauptwerk Du mode d'existence des objets techniques, zu deutsch Die Existenzweise technischer Objekte, von 1958, in welchem er sich - allgemein gesagt - dem Problem der menschlichen Arbeit unter der Bedingung der Anwesenheit technischer Objekte widmet, die zwischen Mensch und Natur situiert sind, als „Mediateur zwischen Mensch und Welt ${ }^{\text {“44 }}$ fungieren und damit in gewisser Weise Medien zwischen beiden Sphären sind.

43 Heilmann 2017.

44 Simondon 2012 [1958], 159. 


\section{Dre-play}

Simondons technikphilosophische Arbeit ist technisch konkret. Er beschreibt Elektronenröhren, Motoren, Turbinen, beschäftigt sich mit Wirkungsgraden, Akkumulatoren, er sinniert über energetische und informationelle Kanäle, Verstärker, Hintergrundrauschen, Kippstufen und Oszillatoren, er kennt auch so technisch exklusive Dinge wie akustische Verzögerungsleitungen, er fokussiert auf Prozesse der Energieumwandlung, schreibt über die materielle Basis von Information, mithin ihre physikalische Verfasstheit und er ist technisch exakt, insofern er beispielsweise zwischen Signal und Impuls unterscheidet. Es geht Simondon dabei nicht um das Wesen der Technik, sondern um die Wesen der Technik, mithin Technikwesen als nicht-menschliche Individuen. „Als Individuen rücken die Maschinenwesen damit neben die psychologischen, soziologischen, biologischen und physikalischen Individuen, und ein wichtiger Teil von Simondons Antwort auf die Frage nach der Technik besteht darin, diesen Individuen in ihrem Nebeneinander zur Anerkennung als Gleichberechtigte $\mathrm{zu}$ verhelfen. “45 Was Simondon dabei auszeichnet, ist, dass obgleich er technische Objekte als Wesen begreift, er sie nicht so untersucht, wie dies beispielsweise die Soziologie, sondern eher wie die Physiologie bzw. Anatomie es mit menschlichen Wesen täte. Er belässt es nicht allein bei einem Studium der äußerlich sichtbaren Erscheinungen, sondern widmet sich im Sinne eines Verständnisses der tatsächlichen stattfindenden Prozesse einer anti-phänomenologischen Anatomie der technischen Wesen und das heißt, er besorgt sie sich und schaut in sie hinein.

Damit ist Simondon techniknäher als andere Technikphilosophen seiner Zeit wie beispielsweise Martin Heidegger, der im November 1953 den Vortrag „Die Frage nach der Technik“ hielt. Für Heidegger liegt das Wesen der Technik im Gestell und so konnte er dem Rheinkraftwerk bescheinigen, Natur zu stellen, insofern diese allein noch Drucklieferant zur Stromproduktion sei. Über die im Kraftwerk befindlichen Turbinen zur Stromerzeugung findet sich bei ihm - anders als bei Simondon - nichts. So wenig wie Heidegger tatsächlich das Kraftwerk am Rhein betrat, genauso wenig öffnete er Artefakte.

Mit techniknahen Ansätzen eint Simondon, dass seine Beschäftigung mit dem Technischen handgreiflich ist. Er besorgt sich die technischen Medien, über die er schreibt und öffnet sie. Er schaut in die Black Box, die bei ihm implizit auf das referiert, was dem Verständnis des technischen Objekts hinderlich ist. Er schreibt: „Der Mensch hat Kenntnis davon, was in die Maschine hineingeht und was aus ihr herauskommt, aber nicht davon, was sich in ihr ereignet: In Gegenwart des Arbeitenden, gewissermaßen vor seinen Augen, vollzieht sich eine Operation, an welcher der Arbeitende aber keinen Anteil hat, selbst wenn er sie steuert oder ihr dient. “46 Über

45 Schmidgen 2012a, 125.

46 Simondon 2012 [1958], 230. Die englische Übersetzung des bereits erwähnten Artikels von Schmid- 
das Versiegeln technischer Artefakte heißt es bei ihm: „Das Versiegeln der anfälligen Organe [eines technischen Objekts, C. B.] markiert diesen Einschnitt zwischen dem Konstrukteur, der sich mit dem Erfinder identifiziert und dem Benutzer, der den Gebrauch des technischen Objekts einzig durch einen ökonomischen Vorgang erwirbt [...].“" ${ }^{47}$ Es geht Simondon folglich nicht um eine Typologie der technischen Objekte, die auf Äußerlichkeit (Boxes) basiert, sondern um eine ,Technogenese‘, die die modulare Entwicklung berücksichtigt und das heißt: die in die Objekte schaut. Simondon fokussiert auf die einzelnen Elemente der technischen Objekte, aber auch auf die Ensembles, in die sie integriert sind, um Netz(werk)e zu formen. Für Simondon gibt es das technische Objekt als sukzessive Entwicklung - also nicht als ein finalistisches Ding, sondern als Trajektorie von Materialisierungen informatischer oder energetischer Schemata, denen eine je spezifische materielle Gestalt zukommt. Programmatisch dafür sind Simondons Fotografien technischer Objekte, die Eingang in seine Analyse finden (s. Abb. 1).

Einer rein phänomenologisch ausgerichteten Technikforschung erteilt Simondon postwendend die Absage. Auf die äußere Erscheinung technischer Objekte dürfe sich eine Untersuchung nicht beschränken - eine implizite Kritik an der Kybernetik - und müsse stattdessen auf die interne (informatorische) Verfasstheit des Technischen fokussieren: „Das Nachdenken über die Automaten ist riskant, denn es läuft Gefahr, sich auf eine Untersuchung der äußeren Eigenschaften zu beschränken, wodurch es eine übermäßige Gleichsetzung vornimmt. Es zählt einzig der Austausch von Energie und Information innerhalb des technischen Objekts oder zwischen dem technischen Objekt und seinem Milieu; die äußeren Verhaltensweisen, die von einem Betrachter gesehen werden, sind keine Gegenstände der wissenschaftlichen Untersuchung. “48 An anderer Stelle schreibt Simondon über die unangebrachte kybernetische Gleichsetzung der Regelung und Nachrichtenübertragung in Lebewesen und Maschinen. Einerseits ähneln beide einander kaum, ${ }^{49}$ andererseits haben viele biologischen Prozesse keine Entsprechung in der Maschine. ${ }^{50}$ Über die kybernetische Analogie von Mensch und Maschine schreibt er daher, „[d]ieser Technizismus ist mehr Phänomenologie als vertiefende Untersuchung [...].“"51

gen trägt daher auch den bezeichnenden Titel „Inside the Black Box. Simondon’s Politics of Technology“ (Schmidgen 2012b).

47 Simondon 2012 [1958], 231.

48 Simondon 2012 [1958], 44.

49 „In Wirklichkeit ähneln die Maschinen dem Menschen kaum, und selbst wenn sie so funktionieren, dass sie vergleichbare Resultate produzieren, ist es sehr selten, dass sie Verfahren einsetzen, die mit denen der Arbeit des individuellen Menschen identisch sind.“ (Simondon 2012 [1958], 71).

50 Die kybernetische Analogie von Lebewesen und Maschine „situiert sich nicht auf der Ebene der Körperfunktionen“, so Simondon (2012 [1958], 127). Es mag trivial anmuten, aber die Materialermüdung einer Maschine ist anderer Natur als die Ermüdung beim Menschen; auch gehen nicht-menschliche Akteure nicht auf Toilette.

51 Simondon 2012 [1958], 136. 

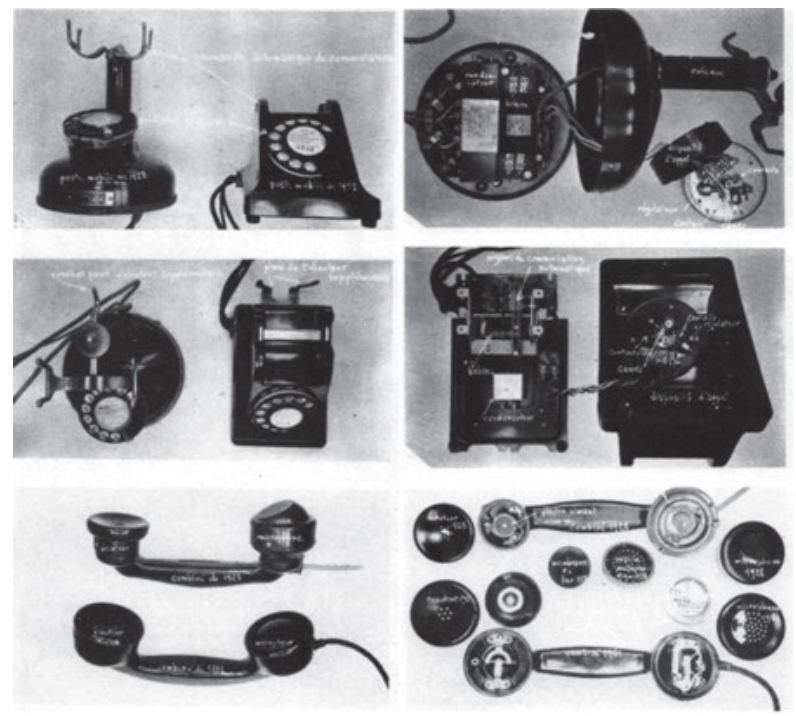

Abb.1: Simondon öffnete zwei Black Boxes: Ein Telefon von 1928 und eines von 1951.

Mit techniknahen Ansätzen eint Simondon, 1.) dass sich technische Objekte für Simondon auf Funktionsschemata zurückführen lassen, ${ }^{52}$ dass 2.) dementsprechend die situierte Anwesenheit einzelner Medien nichts am Bestand des Mediums als Schema ändert und 3.) dass das Technische für ihn eine Bedingung des (sozialen oder wie auch immer gearteten) Handelns ist..$^{53}$ Gleichzeitig aber kontextualisiert er die Maschine in einer Art und Weise, die an die Anthropologie und Soziologie der Technik erinnert, so kommentiert Schmidgen Simondons Technikphilosophie: „Das technische Objekt sieht sich dabei nicht nur in soziale Umfelder eingebettet, sondern auch in technische und natürliche Milieus, die für es spezifisch sind. Die Orientierung an der Maschine als geschlossener Entität wird damit nachhaltig durchbrochen. ${ }^{\text {"54 }} \mathrm{Si}$ mondon interessieren die sozialen, kulturellen, sogar politischen Implikationen und Effekte der Technizität und er nimmt eine soziale und gesellschaftliche Kontextualisierung der technischen Objekte vor, wie sie auch in den Arbeiten von Bruno Latour oder Andrew Pickering anzutreffen sind. Was ihn mit ANT-nahen Ansätzen zudem eint, ist, dass die technischen Objekte bei ihm als Wesen und Individuen auftreten (man könnte auch sagen als nicht-menschliche Akteure), sie damit eine agency innehaben und ihren Beitrag zur Kultur leisten wie auch menschliche Wesen: Die Maschine handelt bei Simondon gleichberechtigt neben dem Menschen, das technische Objekt existiert auf der „gleichen Ebene“ wie der Mensch. ${ }^{55}$

52, „[...] was im technischen Objekt erkannt wird, ist die Form als materielle Kristallisation eines operativen Schemas und eines Denkens, das ein Problem gelöst hat.“ (Simondon 2012 [1958], 229).

53 „Die Technizität ist Teil der Welt, sie ist nicht bloß ein Ensemble von Mitteln, sondern ein Ensemble von Bedingungen des Handelns und Anreizen zum Handeln [...]. “ (Simondon 2012 [1958], 205).

54 Schmidgen 2001, 268-269.

55 Simondon 2012 [1958], 116. 
Allerdings geht Simondon argumentativ und methodisch weiter als die ANT, insofern er einerseits biologische Termini für technische Objekte und ihre Entwicklung verwendet - Genese, Individuum, Hypertelie, Organe -, er andererseits diese Technikwesen obduziert, beziehungsweise technikanatomisch gesprochen: er technische Körper seziert. Es ist oft gesagt worden, dass paradigmatisch für die ANT eigentlich die Bindestriche in ihrem Titel seien, die Akteur-Netzwerk-Theorie, denn in diesen manifestiere sich ihre Fokussierung auf die Netzwerke, die Beziehung zwischen menschlichen und nicht-menschlichen Akteuren, wohingegen Simondon an der Materialität der technischen Objekte festhält. Auch interessieren ihn die Felder, in denen technische Objekte verwirklicht sind, was ihn anschlussfähig für aktuelle medienökologische Forschung macht. Bei aller Mobilität der Medien ${ }^{56}$ heutzutage, ist für ihn die realweltliche Rückbindung von Infrastrukturen für diese kennzeichnend, ihr materielles auf Umgebungen angewiesenes Substrat - auch im Unterschied zu Werkzeugen, so Simondon: „Die Werkzeuge sind frei und abstrakt, sie sind stets überall hin transportierbar; aber die technischen Ensembles sind echte Netze, die konkret an die natürliche Welt gebunden sind; eine Talsperre kann nicht irgendwo erbaut werden, ebenso wenig wie ein Solarschmelzofen." ${ }^{\text {"57 }}$

Auch das Konzept der Operationskette ist bei Simondon implizit angelegt. Das Besondere ist, dass er dieses gemäß seiner Argumentation auf die einzelnen Module eines technischen Objekts bezieht. Operationsketten sind in diesem Sinne nicht allein Handlungsverkettungen zwischen menschlichen und nicht-menschlichen Akteuren, sondern können sich auch intramedial in einem Objekt ausgestalten. Dies entspricht einer mikroskopischen, medienanatomischen Erdung des Begriffs der Operationskette, die eine allzu anthropozentrische Perspektivierung abwendet und nach dem agentiellen Anteil technischer Module innerhalb medientechnischer Prozessarchitekturen fragt. Die Kritik könnte lauten: Der Begriff der Operationskette wurde bisher (man könnte sagen vornehmlich makroskopisch) auf sichtbare und somit ethnologisch nachvollziehbare bzw. erforschbare Handlungsverkettungen bezogen, nicht auf medieninhärente Prozesse. So schreibt Simondon über frühere Motoren, die noch nicht „überdeterminiert“ sind: „Im früheren Motor greift jedes Element an einem bestimmten Moment in der Phase ein und soll dann nicht mehr auf die anderen Elemente einwirken; die Teile des Motors sind wie Personen, die alle arbeiten, wenn sie an der Reihe sind, die sich aber untereinander überhaupt nicht kennen“58 - oder medienpraxeologisch reformuliert handelt es sich hier um eine modulare Ko-Operationskette ohne Konsens der beteiligten Akteure unter Definition eines gemeinsamen Handlungsziels.

56 Thielmann 2014.

57 Simondon 2012 [1958], 203.

58 Simondon 2012 [1958], 21. 
Simondons Verhandlung des Technischen ist modern, was sich auch an dem Status ablesen lässt, den er - im Gegensatz zur Kybernetik stehend - Maschinen zugesteht. Bei Norbert Wiener, dem Gründungsvater der Kybernetik, heißt es im Kontext der Debatte um „Cybernation“599 über die Kybernetisierung des Maschinenparks zur Realisierung einer ,automatic factory“ Folgendes:

In the first place, we can expect an abrupt and final cessation of the demand for the type of factory labor performing purely repetitive tasks. In the long run, the deadly uninteresting nature of the repetitive task may make this a good thing and the source of leisure necessary for man's full cultural development. ${ }^{60}$

Selbstredend sind solche Phantasmas, die an die technische Entwicklung gekoppelt sind, keineswegs neu, sondern wirken selbst 1950 schon antiquiert. Interessant daran ist weniger, was dies für den Menschen bedeute - wie es Wiener expliziert -, sondern was dies zugleich für kybernetische Annahmen über den Status der Maschinen trägt. Denn diese seien, wie Wiener kurz darauf ausführt, nichts weiter als die Sklaven des 20. Jahrhunderts: „Let us remember that the automatic machine [...] is the precise economic equivalent of slave labor."61 Simondon wendet sich gegen solch eine Degradierung bzw. Trivialisierung des Technischen. Bei ihm sind Maschinen keine technischen Sklaven, sondern haben ebenso - um weiterhin politische Begriffe zu bemühen - ein Mitspracherecht. Eines allerdings, das sie selbst nicht artikulieren können, sodass der Technikphilosoph, die Medienwissenschaftlerin usw. - Simondon schreibt vom „Technologen“ bzw. „Mechanologen“ als dem ,Psychologen der Maschinen $^{62}$ - für sie eintreten muss: „Weit entfernt davon, der Aufseher eines Trupps von Sklaven zu sein, ist der Mensch der ständige Organisator einer Gesellschaft der technischen Objekte, die seiner bedürfen, wie Musiker eines Dirigenten bedürfen. “63 Mit der Metapher des Dirigenten des Maschinenorchesters verbindet Simondon auch eine wechselseitige Verfertigung bzw. eine Wechselbeziehung: „Der Dirigent kann die Musiker nur dirigieren, weil er wie diese und mit gleicher Intensität wie diese das aufgeführte Stück spielt: Er mäßigt ihr Tempo oder treibt sie an, aber er wird auch von diesen gemäßigt oder angetrieben. “64 Der Mensch in einer Gesellschaft technischer Objekte ist „mitten unter den Maschinen, die mit ihm handeln und wirken.“65 Das Erkennen dieser wechselseitigen Beziehung des Technischen und Menschlichen ist es, was Simondon anschlussfähig für aktuelle Technik- und Medienforschung macht

59 Hierzu ausführlicher Müggenburg/Pias 2013.

60 Wiener 1950, 161. Die deutsche Übersetzung des Buchs trägt den bezeichnenden Titel Mensch und Menschmaschine.

61 Wiener 1950, 162.

62 Simondon 2012 [1958], 12.

63 Simondon 2012 [1958], 11.

64 Simondon 2012 [1958], 11.

65 Simondon 2012 [1958], 11. 
und zudem das Entweder-Oder - eines technikzentrierten oder anthropologischen Ansatzes - zugunsten einer gegenseitigen Erhellung durchschlägt.

Sind für Bruno Latour technische Dinge „voller Menschen“, ${ }^{66}$ ist dieser Einschreibungsprozess des Menschen in technische Objekte während der Konstruktion auch bei Simondon explizit, der „Mensch ist in den Maschinen durch das Fortbestehen der Erfindung präsent. Was den Maschinen innewohnt, ist menschliche Wirklichkeit, menschliche Geste, die in funktionierenden Strukturen fixiert und kristallisiert ist." ${ }^{67}$ Allerdings entspricht diese Aussage nur auf den ersten Blick einer Exteriorisierungs-These von bspw. Leroi-Gourhan. Die Maschine ist bei Simondon nicht durch Auslagerung menschlicher Tätigkeit gekennzeichnet, auch wird sie nicht schlicht wie ein Werkzeug gebraucht, vielmehr wird sie bedient, gewartet, gereinigt, vernetzt. Die Maschine ist so - im Unterschied zum Werkzeug ${ }^{68}$ - weder eine Prothese (wie programmatisch und prominent vertreten von Ernst Kapp, Grundlinien einer Philosophie der Technik, 1877, Sigmund Freud, Das Unbehagen in der Kultur, 1930 oder Marshall McLuhan, Understanding Media: The Extensions of Man, 1964), noch kann genau umgekehrt der Mensch als Verlängerung der Maschine konzipiert werden. Das technische Objekt begründet durch seine Vernetzung mit anderen technischen Objekten in spezifischen Situationen und Situierungen eine technische Realität, die Teil der menschlichen Kultur ist. Obgleich Simondon keinen eigenen Medienbegriff entfaltet, tritt die Differenz von Werkzeug und technischem Objekt in seinem Werk damit deutlich zutage.

\section{4 reverse}

Wie könnte sich Simondons Methode heutzutage medienhistoriographisch am konkreten Objekt, hier im Falle des Echolots von Alexander Behm, ausgestalten? Dies wäre - wie eingangs erwähnt - als ein epistemologisches Reverse Engineering zu bezeichnen. Zum Prinzip: Das Echolot beruht auf der Aussendung eines akustischen Impulses an Bord eines Schiffes und dem Messen der Zeit bis zum Wiederempfang des am Meeresboden reflektierten Echos dieses Impulses. Bei Kenntnis der exakten Schallgeschwindigkeit im Wasser kann gemäß der Formel Entfernung ist gleich

66 „Die Dinge existieren nicht, ohne voller Menschen zu sein, und je komplexer und moderner diese Dinge sind, desto zahlreicher sind die Menschen, von denen es in ihnen wimmelt.“ (Latour 1996, 37-38). 67 Simondon 2012 [1958], 11.

68 Das Werkzeug tritt bei Simondon nämlich als Verlängerung der menschlichen Geste auf: „das Werkzeug erweitert das Organ und wird von der Geste getragen.“ Das Werkzeug ist ein Objekt, „das es erlaubt, den Körper zu erweitern und dafür zu rüsten, eine Geste zu vollführen“. (Simondon 2012 [1958], 106) Für Simondon ist der Hammer daher ein schlichtes Werkzeug, insofern es auf Materie einwirken kann, er treibt zum Beispiel Spitzeisen in Holz. Instrumente dahingegen „dienen dazu, eine Information zu sammeln, ohne zuvor auf die Welt einzuwirken“. (Simondon 2012 [1958], 106). 
Signalgeschwindigkeit multipliziert mit der vergangenen Zeit, dividiert durch zwei (es handelt sich schließlich um ein Echo) die Meerestiefe errechnet werden $(r=(c \bullet \Delta t): 2)$. Es handelt sich demnach um ein Verfahren der exakten Zeitmessung und damit unterscheidet es sich von bis dato etablierten Verfahren der Tiefenbestimmung, die ausschließlich auf Lotdraht basierten und somit materialaufwendig und bei Tiefseelotungen nicht zeitökonomisch waren. Ist es nach Simondon trügerisch, das technische Objekt über seinen praktischen Nutzen zu definieren, ${ }^{69}$ muss auch beim Echolot betont werden, dass es eigentlich nicht Entfernungen misst (vom Schiff zum Meeresboden), sondern ausschließlich Zeit (das Zeitintervall, $\Delta \mathrm{t}$, zwischen Aussendung und Wiederempfang eines akustischen Impulses). Es ist dieses Zeitintervall, das anschließend erst in eine Raumgröße umgerechnet wird.

Der deutsche Alexander Behm hat das erste funktionale Echolot patentiert und konstruiert. Doch hier beginnen schon die Probleme, denn das Echolot - das heißt das eine - gibt es nicht, genauso wenig wie es das Radio oder den Computer im Kollektivsingular gibt. Was es gibt, sind verschiedene Typen einer Reihe von materiellen Realisierungen eines grundlegenden Schemas, die sich vom Gehäuse, aber auch der inneren Struktur voneinander massiv unterscheiden. Dem epistemologischen RE, dem erkenntnisgerichteten Öffnen der Black Box, vorgeschaltet ist also zunächst eine Selektion, nämlich die Begrenzung der Quellenbasis. Aufgrund der Materiallage entscheide ich mich für das „Behmlot Type 1“, da dies eines der frühesten Echolote ist und mir dessen Bild vorliegt (s. Abb. 2).

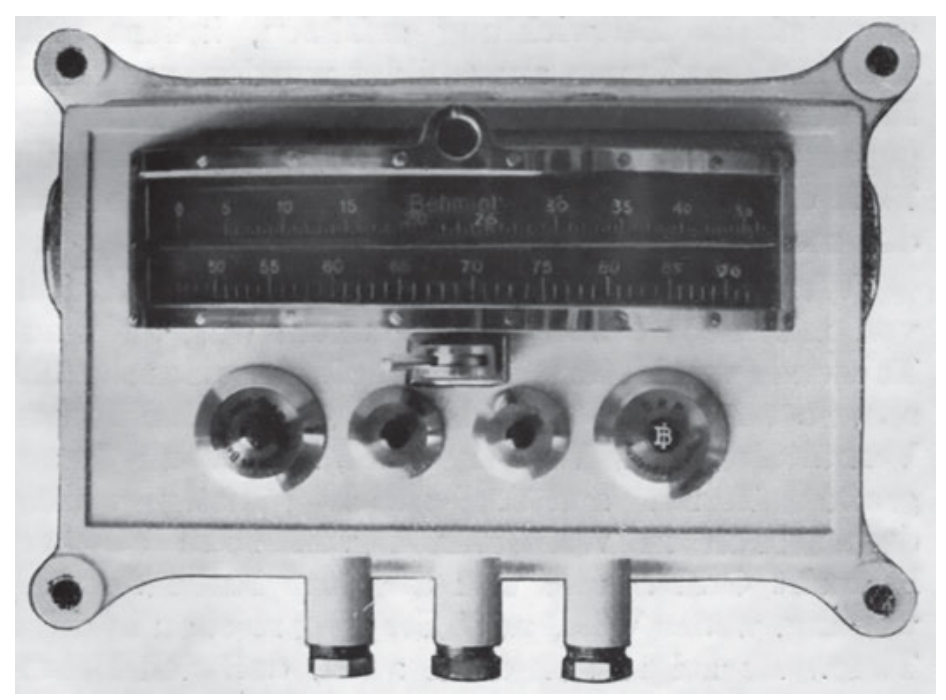

Abb. 2: Das Gehäuse des Behmlots Type 1.

69 Simondon 2012 [1958], 19. 
Als gestaltetes Ding greift das Echolot Type 1 den sich um es herum ausgestalteten Praktiken voraus. Konkret verfügt es über eine Anzeige, auf welcher Meerestiefe direkt abgelesen werden kann, über einen Knopf zum Auslösen des Schallimpulses, einen Knopf zur Hintergrundbeleuchtung der Anzeige und einen Knopf zur Löschung des angezeigten Ergebnisses. Durch den Verwendungskontext des Echolots erübrigen sich dabei ästhetische Fragen weitgehend - anders als beispielsweise beim späteren Fernseh-Möbel, wie Monique Miggelbrink jüngst gezeigt hat. ${ }^{70}$ Das Echolot ist ein technisches Objekt, das von professionalisierten NutzerInnen in einem wissenschaftlich-hydrografischen Kontext gebraucht wird, um Meerestiefen valide und zeitökonomisch zu bestimmen, es muss daher nicht ,hübsch' sein oder sich in eine bestehende Wohnkultur integrieren, es muss schlichtweg funktionieren. Daher erscheint es als ein plumper Kasten - ein Kasten, der keinen Aufschluss über seine Funktionsweise, d. h. die Zeitmessung, gibt, weshalb er entfernt werden muss, um in die Black Box zu schauen (ich muss mich dabei wieder auf eine Fotografie beschränken, die laut der Behm-Echolot-Gesellschaft m.b.H. das Innere des Echolots Type 1 zeigt).

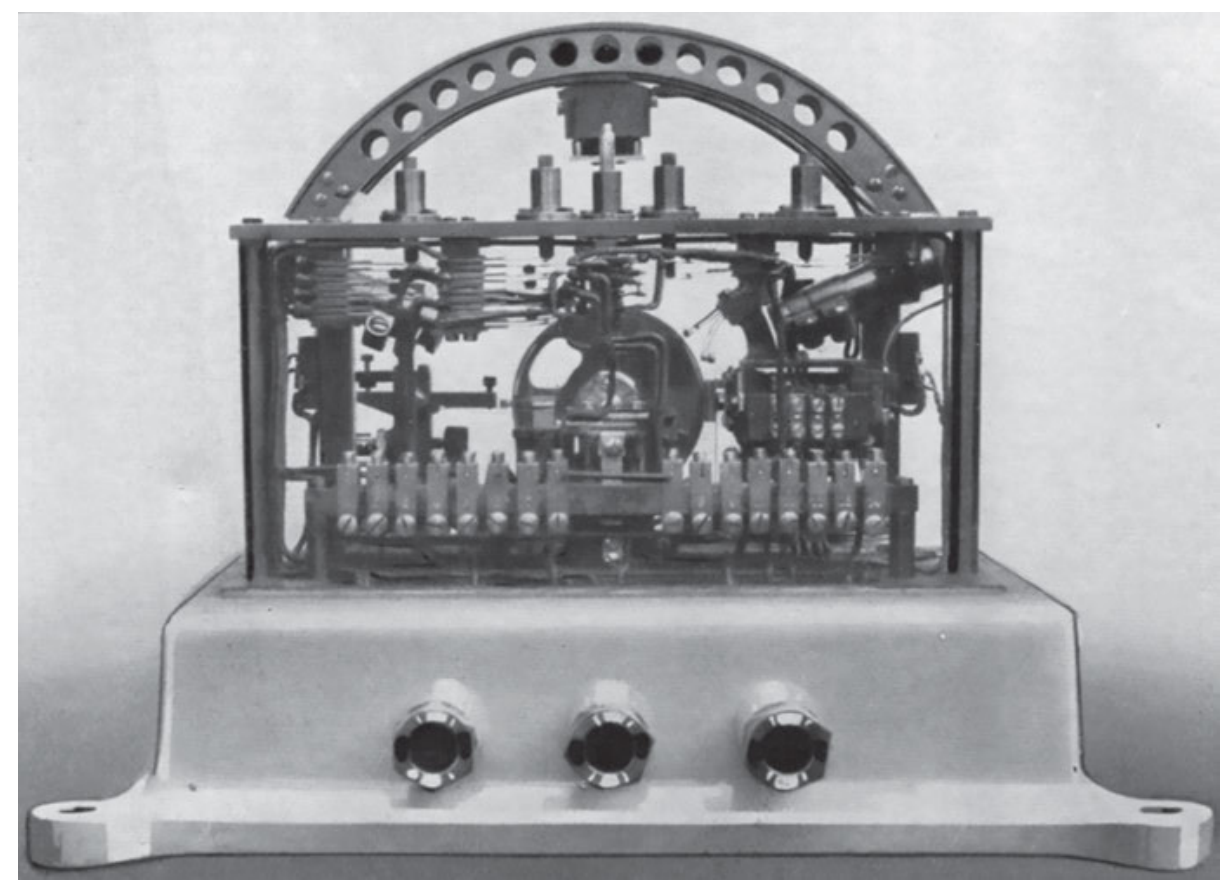

Abb. 3: Das ,Innenleben` des Behmlots Type 1.

70 Miggelbrink 2018. 
Zum Vorschein kommt ein komplexes Gewirr von Drähten, Ankern, Schrauben, Elektromagneten und dergleichen mehr. Mit anderen Worten: Wir sehen, dass das vermeintliche Echolot keineswegs ein kohärentes technisches Objekt ist, sondern in diverse Subsysteme zerfällt wie es Bruno Latour dem Overhead-Projekt bescheinigte: Gerade noch ein Ding, sehen wir uns mit einer Vielzahl von Subdingen konfrontiert. Um im Sinne Simondons aus dem technischen Objekt wieder einen grundlegenden Konstruktionsplan, ein Schema, erstellen zu können, gibt die Fotografie zwar Aufschluss über die innere Verfasstheit des Geräts, aber kaum über seine Prozessarchitektur. Um das epistemologische RE dennoch weiterführen zu können, bedarf es eines historischen Rückschritts, nämlich zu einem Patent und der darin enthaltenen Schematisierung einer idealtypischen Realisierung des Echolots. Konkret handelt es sich um Abbildung 1 von Behms Patent „Kurzzeitmesser“, patentiert im Deutschen Reiche vom 1. Juni 1920 ab, ausgegeben am 18. Januar 1923, Patentschrift Nr. 367202.

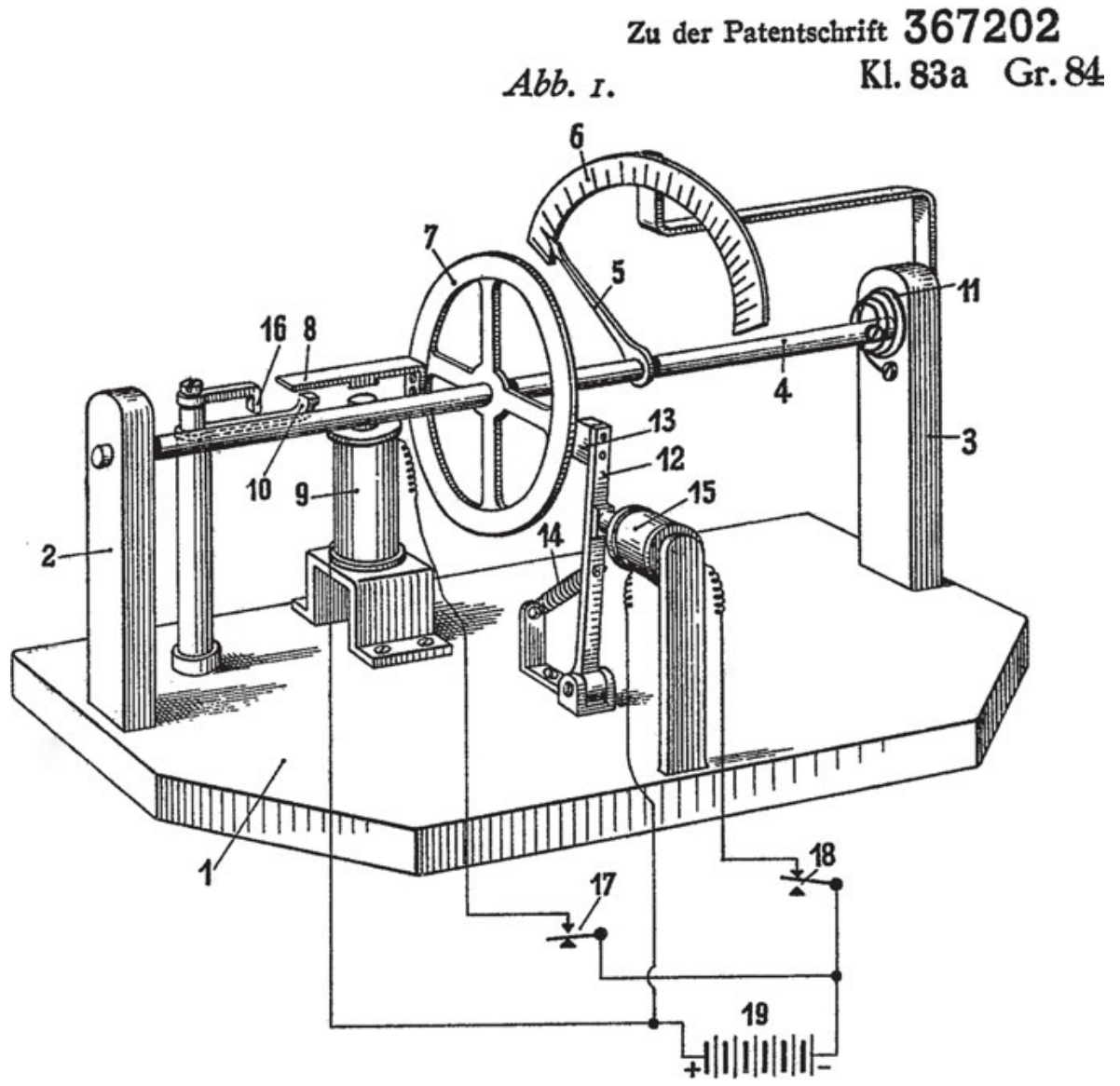

Abb. 4: Prinzip der Zeitmessung des Behmlots Type 1 schematisch dargestellt. 
Diese Schematisierung in Verbindung mit ihrer Erklärung im Patent gibt Aufschluss darüber, worum es sich beim Echolot Type 1 handelt. Die Zeitmessung des Behmlots basiert auf dem Öffnen zweier Stromkreise. Der Sender des akustischen Ortungsimpulses sowie der Empfänger des Echos sind mit jeweils einem Stromkreis der Apparatur verschaltet. Zum exakten Zeitpunkt der Aussendung des Impulses wird ein Stromkreis geöffnet, sodass ein Elektromagnet (9) entmagnetisiert und dadurch ein auf einer Feder (16) gelagerter Hebel (8) abgestoßen wird. Die daraus resultierende Drehung eines Rads (7) ist mit einer Stange (4) mit einem Zeiger (5) gekoppelt, der auf einer Skala (6) einen Ausschlag vollzieht. Durch den Empfang des Echos wird ein zweiter Stromkreis geöffnet, wodurch ein weiterer Elektromagnet (15) entmagnetisiert wird und dadurch eine Bremse (13) die Bewegung des Drehrads stoppt. Entsprechend der Zeit zwischen Impulsaussendung und Wiederempfang hat Zeiger 5 an Skala 6 eine Bewegung vollzogen. Ist die Skala gemäß Schallgeschwindigkeit unter Wasser geeicht, kann Meerestiefe an ihr abgelesen werden.

Wurde oben gesagt, das Echolot messe keine Entfernungen, sondern Zeitintervalle, zeigt sich, dass das Behmlot Type 1 eigentlich auch keine Zeit bestimmt, sondern auf dem Ausschlag eines Zeigers basiert - ein Prinzip, das im 19. Jahrhundert als ,zeitmessender Strom‘ bekannt war und als Zeitmessmethode für kleinste Zeitteile praktiziert wurde. Originär wurde die Logik hinter dem Schema in der Ballistik angewendet, um die Frage nach dem Zeitintervall zwischen Betätigung eines Gewehrabzugs, der Dauer der Entzündung des Schießpulvers und der Dauer des Laufs der Kugel durch ein Gewehr zu klären. Diese Methode geht auf den französischen Physiker Claude Pouillet zurück und machte Dauer durch die Intensität des Ausschlags einer Galvanometernadel errechenbar. ${ }^{71}$ Prominenten Einsatz fand das Verfahren zur Mitte des 19. Jahrhunderts bei Hermann von Helmholtz, der mit diesem erstmals die Ausbreitungsgeschwindigkeit elektrischer Impulse in biologischen Leitern (Nerven) maß und damit das Fühlen und Denken zeitkritisch erdete. ${ }^{72}$ Dies ist relevant für die wissen(schaft)sgeschichtliche Verortung des Echolots Type $1 .^{73}$

Um vor der - wie Schüttpelz schrieb - ,Reifizierung' des Echolots auf seine vorgelagerten Praktiken zu fokussieren, ist notwendigerweise der Konstrukteur Behm selbst entscheidend. Bevor nämlich sein Echolot erst Zeitintervalle bestimmen konnte, musste er bspw. klären, was in der Wissensgeschichte der Akustik seiner Zeit noch eine Leerstelle markierte: Wie gestaltet sich das räumliche Verhalten von Schall unter Wasser, insbesondere seine Reflexion, aus? So Behm: „Meine Arbeit begann mit einer gründlichen Untersuchung der akustischen Verhältnisse im Wasser,

\footnotetext{
71 Pouillet 1844.

72 Helmholtz 1850.

73 Eine vollständigere Wissensgeschichte des Echolots müsste auch einen Diskurs berücksichtigen, in welchem die Laufzeit der Akustik schon vorher zum kritischen Moment avancierte: Die Architekturtheorie und insbesondere die empirisch ausgerichtete Theaterarchitekturforschung im 19. Jahrhundert, siehe bspw. Langhans 1810.
} 
da bis dahin die Existenz eines Echos im Wasser von keiner Seite nachgewiesen war." ${ }^{\text {"74 }}$ Dafür entwickelte er eine experimentalpraktische Messanordnung, die fotografisch Schallreflexionen im Wasser aufzeichnen sollte - übrigens im heimischen Goldfischaquarium, in welchem vormals seine Hausfische schwammen. Der Schallimpuls wurde im Aquarium durch einen Funken ausgelöst, ein zweiter Funke außerhalb diente als Blitzlicht für die Fotografie. Den Strom dazu lieferten zwei so genannte „Leidener Flaschen“ (als studierter Physiker und Elektrotechniker war er mit derlei elektrischen Kondensatoren vertraut), zudem konstruierte Behm einen Doppelschalter, der beide Funken nacheinander, versetzt um eine fünfzehntausendstel Sekunde auslöste (damit der Schall nach $10 \mathrm{~cm}$ Ausbreitung fotografiert werden konnte), was er qua Handfeuerwaffe realisierte. Das so erzeugte Foto stellt zugleich die historisch erste Fotografie einer Schallwelle unter Wasser dar.

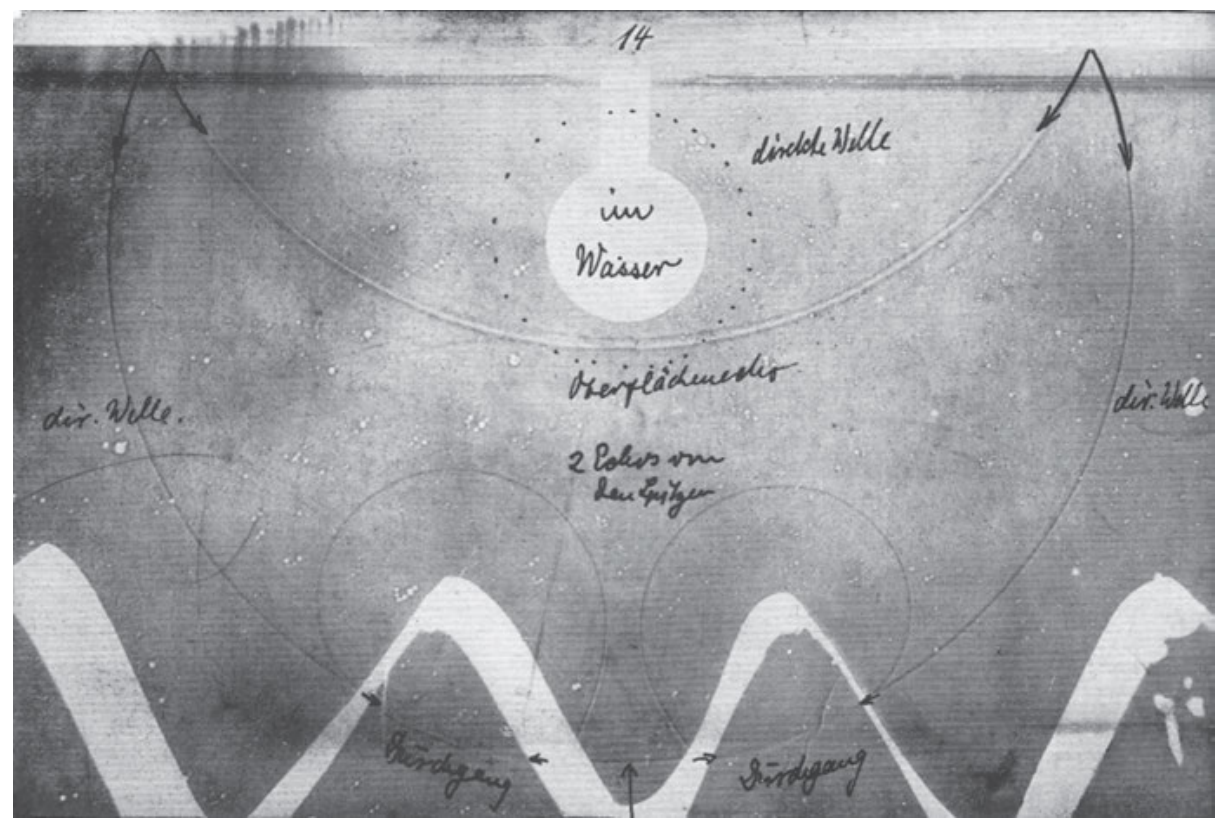

Abb. 5: Auf dem Boden des Aquariums liegt gewelltes Zinkblech, das von der Schallwelle durchdrungen wird. An den Spitzen des Zinkblechs zeigen sich Reflexionen des Schalls.

Bevor also das Echolot Zeitintervalle messen konnte, galt es, eine Anordnung zu konzipieren, die wiederum mikrotemporal operierte, da es galt, unter Wasser einen Knall auszulösen und nur kurze Zeit später (man bedenke die geringe Größe eines Heimaquariums, im Falle Behms $270 \times 250 \times 120 \mathrm{~mm}$, und die Schallgeschwindigkeit unter

74 Behm 1928, 962. 
Wasser von rund 1500 m/s, d. h. 1.500 .000 mm/s) die erste Reflexion des Schallimpulses an den Innenwänden des Aquariums fotografisch zu speichern, um anschließend die Fotografie studieren zu können. Vor dem Medium Echolot stehen in experimentalpraktischer Verschaltung also andere Medien - Fotoapparat -, andere Messanordnungen - zur Klärung von Schallreflexion -, andere technische Objekte - Leidener Flaschen, Pistolen - und nicht zuletzt das elektrotechnische Wissen des Erfinders.

Im Sinne Simondons könnte nun mit fundierter Kenntnis des technischen Objekts und seiner grundlegenden Schematisierung auf seine praktische Verhandlung und auch die Praktiken eingegangen werden, die sich um es herum neu konfigurierten. Das Echolot verkürzte die Dauer einer Lotung, insbesondere bei Tiefseelotungen erheblich. Dauerte eine Tiefsee-Drahtlotung zwischen drei und vier Stunden und brachte ungenaue oder keine Daten und ging zudem meist mit dem Verlust eines Lotgewichts einher (dieses bleib auf dem Meeresboden nachdem auf Grund gestoßen wurde), hatten Echolotungen die Geschwindigkeit von Unterwasserschall. Dies erleichterte in der Praxis das Loten währenddessen die Position des lotenden Schiffes fortwährend neu bestimmt werden musste, was bei stundenlangen Drahtlotungen Komplikationen mit sich brachte. Dahingegen war pro Echolotung nur eine einzige Positionsbestimmung notwendig. Zudem änderte das Echolot das buchstäbliche Bild der Welt, da binnen kürzester Zeit eine enorme Datenfülle (Meerestiefe) erzielt wurde. Während der Deutschen Atlantischen Expedition des Forschungsschiffes Meteor zwischen 1925 und 1927 wurden beispielsweise 60.000 Tiefseelotungen vorgenommen, wohingegen während der britischen Challenger-Expedition zwischen 1872 und 1876 nur rund 400 Tiefsee-Drahtlotungen stattfanden. Dass das Echolot Techniken und Operationsketten der Drahtlotung binnen kürzester Zeit obsolet werden ließ, ist durch seine Praxisvorteile zu erklären: Es war billiger als Drahtlotungen, generierte Daten schneller und war zudem weniger störanfällig während des Lotvorgangs. Tiefe als Funktion von Laufzeit zu bestimmen, war ein Verfahren, dessen praktische Vorteile sich deutlich abzeichneten.

Wo bzw. besser wann nun tritt das Echolot medienhistorisch zutage? In seiner materiellen Kristallisation? In seiner ideellen Schematisierung und damit vor jeder Reifizierung? Im Echolot in seiner historisch frühesten patentierten Formatierung von 1918 (dort operierte es noch chronographisch), wenngleich dieses ein anderes ist als das Echolot in seiner Schematisierung von 1920, wie es sich schließlich durchsetzte? Ist die nach mehreren Prototypen realisierte industrielle Produktion des Echolots, die erst zu seiner Verbreitung führte, entscheidend? Sind nicht die Konstruktion von komplexen Anordnungen zur exakten Zeitmessung, die ja das wesentliche Moment des Echolots sind, zur Mitte des 19. Jahrhunderts die entscheidende Bedingung? Die Antwort ist ein Paradoxon. Sie müsste lauten an all diesen Punkten und an keinem dieser Punkte tritt das Echolot zutage: An keinem dieser Stellen exklusiv, an allen Stellen anteilig. Genau aus diesem Grund schreibt Simondon von der Genese technischer Objekte, der ,Technogenese‘, ihrem sukzessiven Werden, dem Prozess der Individuation bzw. „Konkretisation“ in seiner Gesamtheit: Wie tritt ein technisches Individuum erst 
als ein solches hervor - von einer abstrakten Idee über Prototypen bishin zum realisierten technischen Objekt, welches wiederum ,überdeterminiert“ wird. ${ }^{75}$

Entscheidend sind sicher die Umschlagpunkte von Diskurs in Struktur und vice versa; die Momente, in denen Wissen, Praktiken und Materialien in produktiver Weise zusammentreten, um wiederum materielle Quellen $\mathrm{zu}$ hinterlassen, um die herum sich wiederum Praktiken und medientechnisches Wissen (neu) konfigurieren. Behm jedenfalls mag das Echolot als erster in materielle Form gebracht haben; ,erfunden“ hat er es sicher nicht. Statt als Erfinder erscheint er als gewiefter Finder im medienepistemischen Arsenal seiner Zeit.

\section{Literaturverzeichnis}

Ashby, W. Ross (1956), An Introduction to Cybernetics, London.

Bartz, Christina/Kaerlein, Timo/Miggelbrink, Monique/Neubert, Christoph (2017), „Zur Medialität von Gehäusen. Einleitung“, in: dies. (Hgg.), Gehäuse. Mediale Einkapselungen, Paderborn, 9-23.

Reichspatentamt (1923), Patentschrift Nr.367202, Kl. 83a, Gr. 84 (B94295 IX/83a): Alexander Behm in Kiel. Kurzzeitmesser. Patentiert im Deutschen Reiche vom 1. Juni 1920 ab, ausgegeben am 18. Januar 1923, Berlin. Online vefügbar: http://www.alexanderbehm.de/mediapool/123/1230334/data/1920_Behm-Kurzzeitmesserpatent.pdf (Stand: 18.12.2019).

Behm, Alexander (1928), „Die Entstehung des Echolots“, in: Die Naturwissenschaften 16 (45-47), 962-969.

Bense, Max (1998 [1951]), „Kybernetik oder Die Metatechnik einer Maschine“, in: Elisabeth Walther (Hg.), Max Bense. Ausgewählte Schriften, Bd. 2: Philosophie der Mathematik, Naturwissenschaft und Technik, Stuttgart/Weimar, 429-446.

Bolz, Norbert (1987), „Die Schrift des Films“, in: Friedrich Kittler, Manfred Schneider u. Samuel Weber (Hgg.), Diskursanalysen 1. Medien, Opladen, 26-37.

Borbach, Christoph/Thielmann, Tristan (2019), „Über das Denken in Ko-Operationsketten. Arbeiten am Luftlagebild“, in: Sebastian Gießmann u. Tobias Röhl (Hgg.), Materialität der Kooperation, Wiesbaden, 121-176.

Dewey, John (1988 [1934]), Kunst als Erfahrung (suhrkamp taschenbuch wissenschaft 703), Frankfurt a. M.

Ernst, Wolfgang (2008), „,Merely the Medium‘? Die operative Verschränkung von Logik und Materie“, in: Stefan Münker u. Alexander Roesler (Hgg.), Was ist ein Medium? (suhrkamp taschenbuch wissenschaft 1887), Frankfurt a. M., 158-184.

Gießmann, Sebastian (2018), „Elemente einer Praxistheorie der Medien“, in: Zeitschrift für Medienwissenschaft 19, 95-109.

Hartmann, Frank (2009), Unter die Haut der Welt. Zur Genese des transanthropologischen Raumes, Vortrag am Symposium „Perpektiven einer Anthropologie des Medialen“, Goethe Universität Frankfurt a. M., 30.5.2009, https://homepage.univie.ac.at/frank.hartmann/docs/Hartmann_ Transanthro.pdf (Stand: 13.5.2019).

75 Simondon 2012 [1958], 143. Während der Individuation schreitet die Entwicklung des technischen Objekts nach Simondon von einem metastabilen Zustand zum nächsten voran (Simondon 2012 [1958], 145). 
Heilmann, Till (2016), „Zur Vorgängigkeit der Operationskette in der Medienwissenschaft und bei Leroi-Gourhan“, in: Internationales Jahrbuch für Medienphilosophie 2, 7-29.

Heilmann, Till (2017), „Der Klang der breiten Rille“, in: Internationales Jahrbuch für Medienphilosophie, https://jbmedienphilosophie.de/2017/3-replik-heilmann (Stand: 19.4.2019).

Helmholtz, Hermann von (1850), „Messungen über den zeitlichen Verlauf der Zuckung animalischer Muskeln und die Fortpflanzungsgeschwindigkeit der Reizung in den Nerven“, in: Archiv für Anatomie, Physiologie und wissenschaftliche Medicin 27, 276-364.

Hilgers, Philipp von (2011), „The History of the Black Box. The Clash of a Thing and its Concept“, in: Cultural Politics: An International Journal 7 (1), 41-58.

Jany, Susanne (2015), „Operative Räume. Prozessarchitekturen im späten 19. Jahrhundert“, in: Zeitschrift für Medienwissenschaft 12, 33-43.

Kittler, Friedrich (1986), Grammophon Film Typewriter, Berlin.

Kittler, Friedrich (1990), „Real Time Analysis. Time Axis Manipulation“, in: Michael Scholl u. Georg Christoph Tholen (Hgg.), Zeit-Zeichen. Aufschübe und Interferenzen zwischen Endzeit und Echtzeit, Weinheim, 363-377.

Langhans, Carl Ferdinand (1810), Ueber Theater oder Bemerkungen über Katakustik in Bezug auf Theater, Berlin.

Latour, Bruno (1996 [1991]), Der Berliner Schlüssel. Erkundungen eines Liebhabers der Wissenschaften, Berlin. - ders. „Inscrire dans la nature des choses ou la clef berlinoise“, in: Alliage 1991 (6), 4-16.

Latour, Bruno (1999), Pandora's Hope. Essays on the Reality of Science Studies, Cambridge (MA).

Leroi-Gourhan, André (1980 [1964-1965]), Hand und Wort. Die Evolution von Technik, Sprache und Kunst (suhrkamp taschenbuch wissenschaft 700), Frankfurt a. M. - ders. (1964-1965), Le geste et la parole: Technique et langage \& La mémoire et les rythmes. 2 Bde., Paris.

Light, Jennifer S. (1999), „When Computers Were Women“, in: Technology and Culture 40 (3), 455-483.

Maresch, Rudolf (1996), „Blindflug des Geistes. Was heißt (technische) Medientheorie?“, in: Telepolis: Magazin 8.7.1996, https://heise.de/-3412557 (Stand 17.4.2019).

Mauss, Marcel (1947), Manuel d'ethnographie, Paris.

Maye, Harun (2010), „Was ist eine Kulturtechnik?“, in: Zeitschrift für Medien- und Kulturforschung 1 (1), 121-135.

Miggelbrink, Monique (2018), Fernsehen und Wohnkultur. Zur Vermöbelung von Fernsehgeräten in der BRD der 1950er- und 1960er-Jahre, Bielfeld.

Müggenburg, Jan/Pias, Claus (2013), „Blöde Sklaven oder lebhafte Artefakte? Eine Debatte der 1960er“, in: Hannelore Bublitz, Irina Kaldrack, Theo Röhle u. Mirna Zeman (Hgg.), Automatismen - Selbst-Technologien, Paderborn, 45-69.

Pias, Claus (2004), „Elektronenhirn und verbotene Zone. Zur kybernetischen Ökonomie des Digitalen“, in: Alexander Böhnke u. Jens Schröter (Hgg.), Analog/Digital - Opposition oder Kontinuum?, Bielefeld, 295-310.

Pouillet, Claude (1844), „Note sur un moyen de mesurer des intervalles de temps extrément courts, comme la durée du choc des corps élastiques, celle du débandement des ressorts, de l'inflammation de la poudre, etc.", in: Comptes rendus hebdomadaires des séances de l'Académie des sciences 19, 1384-1389.

Schmidgen, Henning (2001), „Der Psychologe der Maschinen. Über Gilbert Simondon und zwei Theorien technischer Objekte“, in: Christiane Kraft Alsop (Hg.), Grenzgängerin/Bridges between Disciplines: Festschrift für Irmingard Staeuble, Heidelberg/Kröning, 265-287.

Schmidgen, Henning (2012a), „Das Konzert der Maschinen. Simondons politisches Programm“, in: Zeitschrift für Medien- und Kulturforschung 3 (2), 117-134. 
Schmidgen, Henning (2012b), „Inside the Black Box. Simondon's Politics of Technology“, in:

SubStance 41 (3), 16-31.

Schüttpelz, Erhard (2006), „Die medienanthropologische Kehre der Kulturtechniken“, in: Archiv für Mediengeschichte 6, 87-110.

Schüttpelz, Erhard (2010), „Körpertechniken“, in: Zeitschrift für Medien- und Kulturforschung 1 (1), 101-120.

Simondon, Gilbert (2012 [1958]), Die Existenzweise technischer Objekte, Zürich.

Thielmann, Tristan (2012), „Taking into Account. Harold Garfinkels Beitrag zu einer Theorie sozialer Medien“, in: Zeitschrift für Medienwissenschaft 6, 85-102.

Thielmann, Tristan/Schüttpelz, Erhard (Hgg.) (2013), Akteur-Medien-Theorie, Bielefeld.

Thielmann, Tristan (2014), „Mobile Medien“, in: Jens Schröter (Hg.), Handbuch Medienwissenschaft, Stuttgart, 350-359.

Trenkle, Fritz (1979), Die deutschen Funkmeßverfahren bis 1945, Stuttgart.

Trenkle, Fritz (1982a), Die deutschen Funkpeil- und -Horch-Verfahren bis 1945, Frankfurt a. M.

Trenkle, Fritz (1982b), Die deutschen Funkstörverfahren bis 1945, Frankfurt a. M.

Trenkle, Fritz (1987), Die deutschen Funklenkverfahren bis 1945, Heidelberg.

Weber, Heike (2017), „Black Boxing? Zur Vermittlung von Konsumtechniken über Gehäuse- und Schnittstellendesign“, in: Christina Bartz, Timo Kaerlein, Monique Miggelbrink u. Christoph Neubert (Hgg), Gehäuse. Mediale Einkapselungen, Paderborn, 115-136.

Wiener, Norbert (1950), The Human Use of Human Beings, Boston.

Winkler, Hartmut (1999), „Die prekäre Rolle der Technik. Technikzentrierte versus ,anthropologische“ Mediengeschichtsschreibung “, in: Claus Pias (Hg.), [me'dian]'. dreizehn vortraege zur medienkultur, Weimar, 221-238.

Winkler, Hartmut (2015), Prozessieren. Die dritte, vernachlässigte Medienfunktion, Paderborn.

\section{Bildnachweise}

Abb.1: Simondon 2012 (1956), $264 \& 265$.

Abb. 2: Behm 1928, 965.

Abb. 3: Behm 1928, 966.

Abb. 4: Reichspatentamt 1923, 5.

Abb. 5: Behm 1928, 964. 
